\title{
Innovative Solution for Grasping Forceps Support Suction to Facilitate Uniportal Video-Assisted Thoracoscopic Surgery
}

\author{
Xugang Zhang ${ }^{1, *}$ Lei Yang ${ }^{2, *}$ Jianrui Wang ${ }^{3}$ Bo Wei ${ }^{1}$ \\ ${ }^{1}$ Department of Thoracic Surgery, Beijing Shijitan Hospital, Capital \\ Medical University, Beijing, People's Republic of China \\ 2 Department of Radiology, Beijing Shijitan Hospital, Capital Medical \\ University, Beijing, People's Republic of China \\ ${ }^{3}$ Department of Thoracic Surgery, The Fourth Hospital of Yulin, \\ Shanxi Provice, People's Republic of China
}

Both authors contributed equally to this work.

Thorac Cardiovasc Surg 2019;67:151-154.

\author{
Address for correspondence Bo Wei, MD, PhD, Department of \\ Thoracic Surgery, Beijing Shijitan Hospital, Capital Medical University, \\ Beijing 100038, People's Republic of China \\ (e-mail: weibos@126.com).
}

\begin{abstract}
Keywords

- VATS

- instrument

- surgical technique

Uniportal video-assisted thoracoscopic surgery has been limited to the use of very few instruments, which but at least include energy device, grasping forceps, and suction. The last two instruments must be temporarily replaced by each other in response to situation. This step prolongs operative duration and potentially impairs the surgical efficiency. We describe a novel instrument design of grasping forceps support suction. Its continuous suction can clear the smoke and blood simultaneously to keep the operative field dry and clean, minimize the requirement to exchange surgical devices, and allow for optimized surgical workflow.
\end{abstract}

\section{Introduction}

Surgeons apply grasping forceps and suction in minimally invasive surgical environments to cooperate with the energy device that handled in right hand. ${ }^{1}$ However, unlike in minithoracotomy or even multiport video-assisted thoracoscopic surgery (VATS), where access is readily available or instrument motion is relatively unrestricted, the use of these instruments in uniport VATS has been limited by the single surgical corridors. The two devices possibly interact with each other when they are maneuvered, and must be temporarily removed and replaced during surgery ( - Fig. $\mathbf{1}$ ).

Such frequent instrument exchange during endoscopic surgery has several shortcomings: (1) it is time-consuming; (2) it disrupts the flow of the procedure, and can break the concentration of the surgeon; (3) it poses a safety risk to the patient. Due to the limited view obtained with most endoscopes, there may be instances during an insertion or removal when the instrument cannot be seen while it is still inside the patient. These "blind" episodes are potential

received

May 3, 2018

accepted after revision

June 14, 2018

published online

July 18, 2018 causes of trauma that can be minimized by reducing the frequency of instrument exchanges. ${ }^{2,3}$

With the aim of solving this problem, we developed a novel instrument of grasping forceps support suction. It minimized the requirement to exchange surgical devices when performing uniportal VATS, and allowed for new maneuvers that optimized surgical workflow, such as the ability to ensure continuous suction during surgery, maintaining a dry surgical field and an improved endoscopic visibility.

\section{Technique}

\section{Structure of the Grasping Forceps Support Suction}

Our device (-Fig. 2) was adapted from standard grasping forceps originally developed for target tissue holding that were augmented to co-function as lymph node grippers. The suction tube, which is taken from a disposal vein infusion set, accompanied the shaft of the instrument, and then the aspiration system is connected to the handle. As such the
DOI https://doi.org/ 10.1055/s-0038-1667143. ISSN 0171-6425. (c) 2019 Georg Thieme Verlag KC Stuttgart · New York 

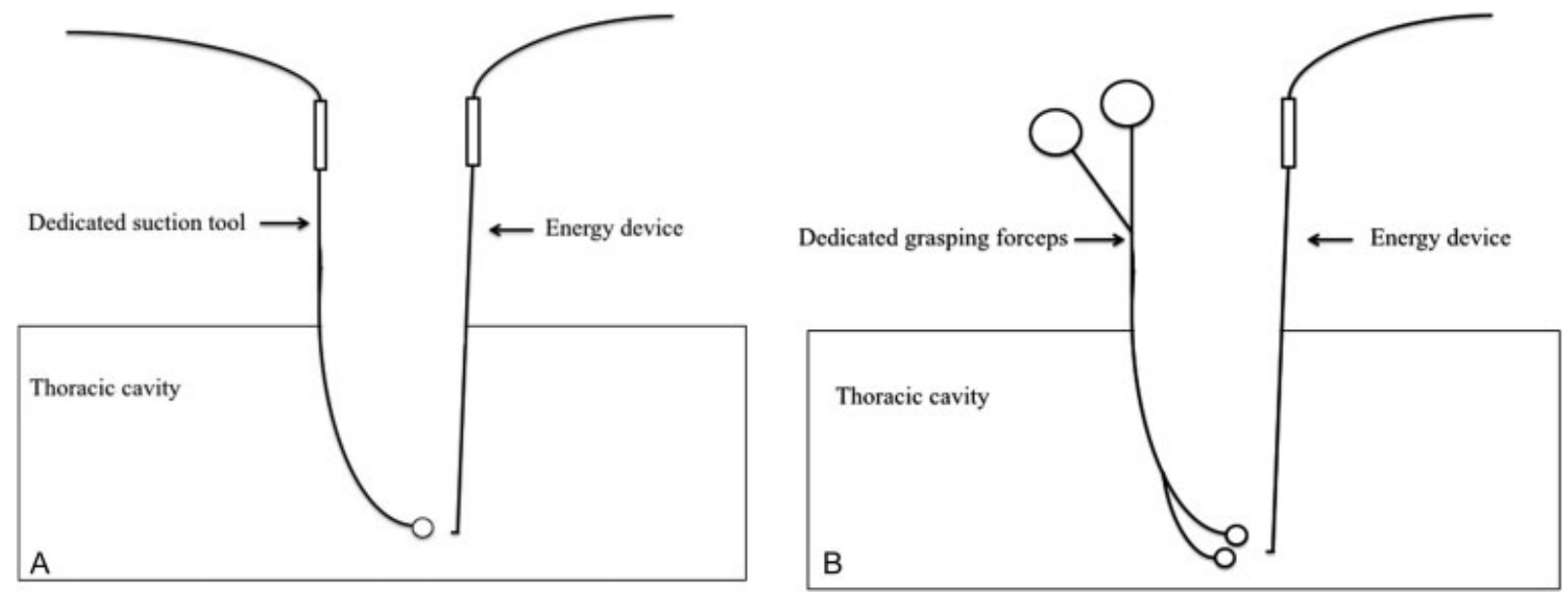

Fig. 1 The drawing represents the major manipulated pattern of current uniportal video-assisted thoracoscopic surgery. It is a standard twohanded surgery, and the surgeon is limited to using only one additional surgical tool when using a dedicated energy device in right hand for dissection. Primary surgical instruments in left hand-including suction devices (A) and grasping forceps (B)-must be switched frequently in response to situation.

instrument tip does additionally function as an aspirator while it still serves its original function as gripper.

This device works identically to both a standard clamp forceps and a dedicated suction. When the clamp grasps or handles the target tissue, the continuous suction permits an improved endoscopic visualization via blood, smoke, and other cautery by-products synchronized cleaning.

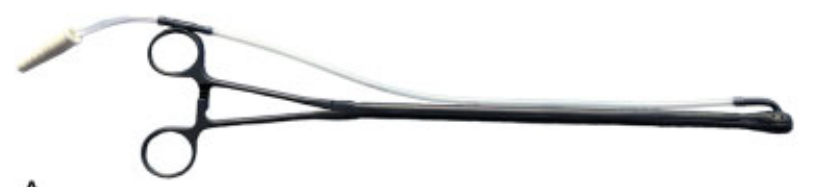

A
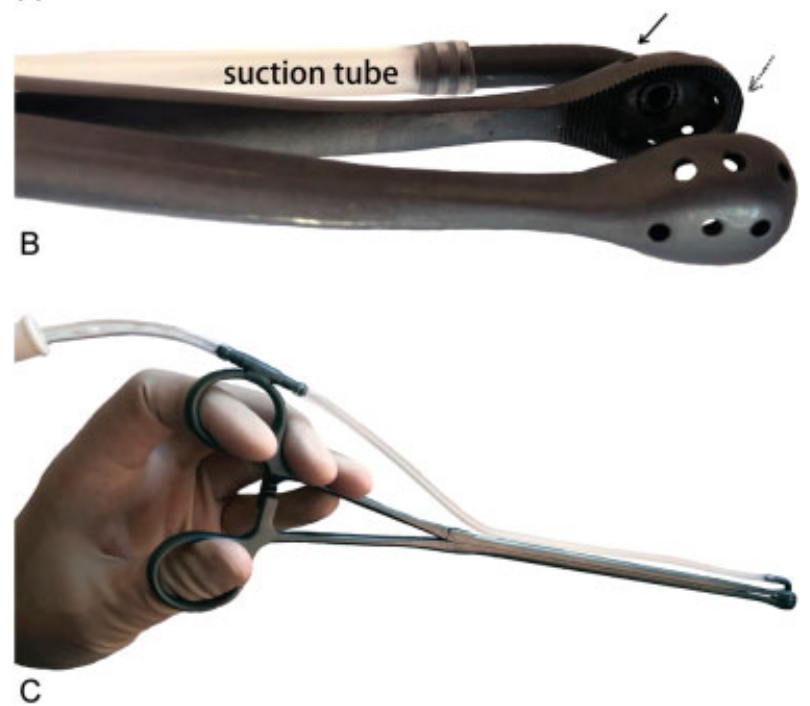

Fig. 2 A panoramic view of the grasping forceps support suction (A). (B) Distal tip of the forceps connected with suction tube (solid arrow). The pliers had serrations to improve the grip (dashed arrow). When the instrument grasps or handles the target tissue, its suction is still working to clear smoke and blood, thereby keeping the surgical field dry and clean. (C) Please note the handle manner of the instrument.

\section{Clinical Experience}

After its 6 months clinical application, our experience demonstrated that the combinations of two instruments provided maximal efficiency in workflow and reduced the requirements to remove and reinsert surgical devices when suction was intermittently needed.

\section{Discussion}

Improved efficiency in the performance of a procedure is one of the primary goals of instrument design. ${ }^{2}$ Multifunctionality clearly plays a role in making an instrument more effective and hence more popular among surgeons. ${ }^{2,3}$ Solely improving the primary function of an instrument at the detriment of secondary and tertiary uses decreases its flexibility and could therefore limit its clinical popularizing. ${ }^{2} \mathrm{By}$ somehow combining the function of two of the main devices, the number of device changes necessary to complete the procedure could be reduced. This would potentially heighten the surgical effectiveness, shorten the operative duration, and lessen the underlying trauma to the patient during an instrument exchange. This is more important in uniportal VATS due to its very limited access.

Attending to the combination of a grasping forceps and a suction device, our newly developed instrument works as of dual-function, it offers several advantages compared with what they currently are. Namely, it potentially increases surgical efficiency and enhances workflow by expanding the functions of standard clamping forceps to dually serve as suction apparatus. It allows a simultaneous clearing of smoke and blood resulting from electrocautery during surgery. Meanwhile, it also allows an increased surgical efficiency by minimizing the repetitive removal/insertion of instruments, thereby reducing the risk of interfering with each other, and potentially reducing operative duration. Finally, it reduces the reliance on assistant(s) and increases 
surgical proficiency. These advantages render this novel, dual-functional, minimal-access device has the potential to make a substantial contribution to uniportal VATS, minimally invasive or traditional open surgery.

\section{Disclaimer}

The German Society for Thoracic and Cardiovascular Surgery (DGTHG) and The Thoracic and Cardiovascular Surgeon neither endorse nor discourage the use of the new technology described in this publication.

\section{Conflict of Interest}

The patent of this instrument is waived to facilitate its clinical distribution.

\section{Acknowledgments}

This study is supported by Special Funds for the Construction of High Level Health Technical Personnel in Beijing and Beijing Municipal Administrated Hospital Incubating Program, code: PX2016044. The authors had full control of the study design, methods used, outcome measurements, analysis of data, and production of the written report.

Xugang Zhang MD, PhD and Lei Yang MD have made a great deal of contribution to instrument design, testing, and usage.

\section{References}

1 Gonzalez-Rivas D, Sihoe ADL. Important technical details during uniportal video-assisted thoracoscopic major resections. Thorac Surg Clin 2017;27(04):357-372

2 Mehta NY, Haluck RS, Frecker MI, Snyder AJ. Sequence and task analysis of instrument use in common laparoscopic procedures. Surg Endosc 2002;16(02):280-285

3 Mittelstein D, Deng J, Kohan R, Sadeghi M, Maarek JM, Zada G. Novel technique of a multifunctional electrosurgical system for minimally invasive surgery. J Neurosurg 2017;126(03):997-1002 